\title{
Avaliação da Adequação do Controle de Fatores de Risco Cardiovascular após Cirurgia de Revascularização Miocárdica
}

\author{
Evaluating the Adequacy of Cardiovascular Risk Factor Control after Myocardial Revascularization Surgery \\ Andrea Cristina Costa Barbosa, Luiz Carlos Santana Passos, Antônio Alberto Lopes, Wanewman Lins Guedes de \\ Andrade, Luciano Rapold Souza, Maurício Batista Nunes \\ Universidade Federal da Bahia, Hospital Português, Bahia, BA - Brasil
}

\section{Resumo}

Fundamento: Os fatores de risco cardiovascular (FR) são responsáveis pela ocorrência de eventos cardiovasculares.

Objetivo: Estimar o porcentual de pacientes submetidos a cirurgia de revascularização miocárdica (RM) que conseguem controle adequado de fatores de risco (FR) modificáveis, pelo menos seis meses após o procedimento.

Métodos: O estudo incluiu 88 pacientes, no qual se realizaram análise de prontuários e entrevista clínica, entre seis e 12 meses após a realização de cirurgia de RM em hospital de referência para doenças cardiovasculares, no período de janeiro a dezembro de 2004.

Resultados: A média de idade foi 63,1 $\pm 9,9$ anos: 51 (58\%) eram do sexo masculino, 86 (97,7\%), hipertensos, 38 (43,2\%), diabéticos, 85 (96,6\%), dislipidêmicos e 10 (11,4\%), tabagistas. O controle da hipertensão (PA $<140 \times 90 \mathrm{mmHg}$ ) foi atingido em $24,4 \%$ dos pacientes. Para o colesterol (colesterol $\mathrm{LDL}<100 \mathrm{mg} / \mathrm{dl}$ ) e para o diabete melito (glicemia $<110$ ), os níveis de controle foram, respectivamente, $30,6 \%$ e $31,6 \%$. O uso de anti-hipertensivos, agentes hipoglicemiantes (orais ou insulina) e estatinas, quando indicado, foi, respectivamente, 96,5\%, 92,1\%, 78,8\%. Entretanto, analisando-se a tríade hipertensão, diabete e hipercolesterolemia, apenas $\mathbf{1 4 , 8} \%$ do total de pacientes apresentavam níveis de pressão arterial, glicemia e colesterol LDL dentro dos limites aceitáveis.

Conclusão: Apesar do uso freqüente de medicações para controle da hipertensão, diabete e hipercolesterolemia, o controle de fatores de risco ainda é realizado de forma insuficiente nos pacientes revascularizados, o que sugere grande potencial para a melhoria da prática clínica. (Arq Bras Cardiol 2007;89(6):362-369)

Palavras-chave: Medição de risco, revascularização miocárdica, prevenção de doenças, controle.

Summary

Background: Cardiovascular risk factors (RF) for coronary artery disease (CAD) are responsible for the occurrence of cardiovascular events.

Objective: To estimate the percentage of patients submitted to myocardial revascularization surgery (MRS) that attains adequate control of modifiable RF at least six months after the surgery.

Methods: Data collection was based on the review of medical records and a clinical interview of 88 patients submitted to MRS between January and December of 2004 at a reference hospital for cardiovascular diseases. The patients were interviewed in the follow-up period, between six and twelve months after the surgical revascularization.

Results: Mean age was $63.1 \pm 9.9$ years; 51 patients (58\%) were males, 86 (97.7\%) were hypertensive, 38 (43.2\%) were diabetic, 85 (96.6\%) had hypercholesterolemia and 10 (11.4\%) were smokers. Hypertension control (PA < $140 \times 90 \mathrm{mmHg}$ ) was attained by 24.4\% of the patients; cholesterol (LDL cholesterol $<100 \mathrm{mg} / \mathrm{dl}$ ) and diabetes control (blood glucose levels $<110 \mathrm{mg} / \mathrm{dl}$ ) levels were $30.6 \%$ and $31.6 \%$, respectively. The use of antihypertensive drugs, hypoglycemic agents and statins, when indicated, were $96.5 \%, 92.1 \%$ and $78.8 \%$, respectively. However, only $14.8 \%$ patients had their blood pressure, glucose and cholesterol levels within the limits accepted as adequate control.

Conclusion: Despite the frequent use of drugs to control hypertension, diabetes and hypercholesterolemia, a high proportion of patients still do not achieve the target levels of risk factor control recommended by current guidelines at least six months after revascularization surgery, which suggests there is a great potential for improvement in clinical practice. (Arq Bras Cardiol 2007;89(6):327-333)

Key words: Risk assessment; myocardial revascularization; disease prevention; control.

Full texts in English - http://www.arquivosonline.com.br

Correspondência: Andrea Cristina Costa Barbosa •

Rua São Paulo, 218/203 - Pituba - 41830-181 - Salvador, BA - Brasil

E-mail: andreabarbosa@cardiol.br

Artigo recebido em 03/03/07; revisado recebido em 05/07/07; aceito em 16/08/07. 


\section{Artigo Original}

\section{Introdução}

A cirurgia de revascularização miocárdica (RM), procedimento invasivo de elevada complexidade para o tratamento da aterosclerose coronariana, promove o alívio dos sintomas, melhora a tolerância aos esforços físicos e aumenta a sobrevida nos casos mais graves da doença ${ }^{1}$. Com o tempo, contudo, esses pacientes podem apresentar progressão da aterosclerose tanto nas artérias nativas quanto nos enxertos, dado que a cirurgia exerce apenas ação mecânica de bypass sobre as placas de aterosclerose, não atuando nos fatores etiopatogênicos da doença. Portanto, a implementação de ações preventivas dirigidas para o controle de fatores de risco (FR) relacionados ao desenvolvimento de doença arterial coronariana (DAC) pode não somente evitar novos eventos isquêmicos agudos, como promover a regressão de placas de aterosclerose já existentes ${ }^{2-4}$.

Entre 1995 e 1996, a Sociedade Européia de Cardiologia realizou um estudo denominado EUROASPIRE ${ }^{5}$, que avaliou o controle de FR modificáveis entre pacientes portadores de doença coronariana estabelecida. Após a publicação de nova diretriz em 1998 para prevenção secundária em pacientes coronarianos, com pontos de corte menores para o controle da dislipidemia, diabete e hipertensão ${ }^{6}$, nova avaliação foi realizada. No EUROASPIRE $\mathrm{II}^{7}$, todavia, não foram observadas melhorias na prática de medidas preventivas após cirurgia de RM. No Brasil, não existem estudos recentes avaliando o controle da tríade maligna hipertensão, diabete e dislipidemia após cirurgia de RM depois da publicação das últimas diretrizes da Sociedade Brasileira de Cardiologia, com recomendações mais atuais para o controle de $\mathrm{FR}^{8-10}$.

O objetivo deste estudo é avaliar a adequação do controle dos principais FR à luz das mais recentes recomendações pelas diretrizes entre pacientes submetidos a cirurgia de RM.

\section{Métodos}

Trata-se de estudo observacional ambispectivo envolvendo pacientes submetidos a cirurgia de RM em hospital filantrópico da cidade de Salvador, o Hospital Português. Esse hospital possui oito leitos de terapia intensiva exclusivos para o cuidado pós-operatório de pacientes submetidos a cirurgias cardiovasculares.

Todos os pacientes submetidos a cirurgia de RM no ano de 2004 e que receberam alta hospitalar foram considerados elegíveis para o estudo. Dos 142 pacientes revascularizados entre janeiro e dezembro de 2004, 12 faleceram na fase intra-hospitalar. Dos 130 pacientes que receberam alta, quatro $(3,1 \%)$ faleceram antes do contato telefônico, 19 não foram localizados por inconsistências no número de telefone e endereço constantes do cadastro hospitalar (14,6\%), 17 não participaram do estudo pela impossibilidade de comparecer à entrevista clínica por problemas financeiros $(13,1 \%)$, e apenas dois se recusaram a participar do estudo (1,5\%). Portanto, 88 pacientes foram contatados e aceitaram participar da pesquisa (82,2\% de taxa de participação ajustada, ou seja, porcentagem de participação entre os sobreviventes que foram contatados). Após contato telefônico, foram agendadas entrevistas clínicas para obtenção de consentimento informado por escrito e informações complementares.
Dados referentes à internação foram coletados de forma retrospectiva a partir da análise dos prontuários médicos, de forma sistemática, por meio de formulário padrão contendo informações relativas a variáveis de identificação, como peso, diagnóstico que levou ao procedimento, seguro de saúde, uso prévio de medicações, presença de FR declarados na admissão (hipertensão, diabete melito, hipercolesterolemia, tabagismo e antecedentes familiares de coronariopatia), número de enxertos realizados, uso de balão intra-aórtico, circulação extracorpórea (CEC), tempo de CEC e número de dias de internação em UTI.

Foram considerados hipertensos todos os pacientes que se declaravam com esse diagnóstico ou que fizessem uso de medicações anti-hipertensivas. De forma idêntica, foi considerada para o diagnóstico de diabete a declaração desse diagnóstico pelo paciente ou o uso de antidiabéticos orais ou insulina; e para o diagnóstico de dislipidemia, a sua referência ou o uso de medicação redutora do colesterol.

O período decorrido entre a cirurgia e a entrevista clínica variou entre seis meses e um ano. Na entrevista foram confirmadas informações referentes à presença de $F R$, além de grau de escolaridade, renda familiar em número de salários mínimos, número de avaliações com os respectivos médicos assistentes após a cirurgia, e uso de medicações de forma regular no momento da entrevista. Os pacientes também foram questionados quanto ao hábito de fumar, se praticavam atividade física (considerado positivo se o paciente fizesse no mínimo 30 minutos de atividade aeróbica contínua três vezes por semana) e sobre o seu peso atual. No momento da entrevista foi aferida a pressão arterial de acordo com as recomendações da $\vee$ Diretriz Brasileira de Hipertensão Arterial. Definiu-se como hipertensão controlada quando níveis pressóricos inferiores a 140×90 mmHg (ou 130x80 mmHg se diabético) eram atingidos ${ }^{8}$. A circunferência abdominal foi obtida conforme preconizado pala I Diretriz Brasileira de Diagnóstico e Tratamento da Síndrome Metabólica ${ }^{9}$, sendo considerada ideal circunferência inferior a $90 \mathrm{~cm}$ para homens e $80 \mathrm{~cm}$ para mulheres, de acordo com os critérios de obesidade abdominal para a América do Sul preconizados pela International Diabetes Federation (IDF) ${ }^{11}$. O cálculo do Índice de massa corpórea (IMC) foi feito baseando-se no peso e na altura referidos pelos pacientes, sendo considerado sobrepeso um IMC $\geq 25 \mathrm{~kg} / \mathrm{m}^{2}$, e obesidade um IMC $\geq 30 \mathrm{~kg} / \mathrm{m}^{2}$. Para a avaliação do perfil lipídico (colesterol total, colesterol LDL, colesterol $\mathrm{HDL}$, triglicérides) e do diabete (glicemia de jejum) foram considerados os resultados de exames realizados nos últimos quatro meses precedentes à entrevista. Em relação ao controle da hipercolesterolemia foram consideradas as recomendações das III Diretrizes Brasileiras Sobre Dislipidemias (colesterol total $<200, \mathrm{LDL}<100 \mathrm{mg} / \mathrm{dl}, \mathrm{HDL}>40 \mathrm{mg} / \mathrm{dl}$ ou $>45 \mathrm{mg} / \mathrm{dl}$ para diabéticos, e triglicérides $<150 \mathrm{mg} / \mathrm{dl})^{10}$. Em relação ao diabete, foi considerado controle adequado quando o seu valor era inferior a $110 \mathrm{mg} / \mathrm{dl}$, de acordo com o nível de cuidados mínimos das diretrizes da IDF ${ }^{11}$. Para o diagnóstico da síndrome metabólica, foram utilizados os critérios da I Diretriz Brasileira de Diagnóstico e Tratamento da Síndrome Metabólica9 .

Esse trabalho foi aprovado pelo Comitê de Ética em Pesquisa do Hospital Português, e os procedimentos de coleta de dados e entrevistas foram conduzidos de acordo com os princípios da Declaração de Helsinki. 
As variáveis contínuas foram descritas por meio de média, desvio padrão e mediana, e comparadas pelo teste de Mann-Whitney, exceto para idade entre homens e mulheres, e número de consultas médicas entre pacientes usuários de convênios privados de saúde e pacientes atendidos pelo SUS, que foram considerados apresentando distribuição normal e comparados por meio do teste $t$ de Student para amostras não-pareadas. As variáveis categóricas foram descritas pelas distribuições de freqüências absolutas e relativas, sendo utilizado o teste do $\chi^{2}$ ou teste exato de Fisher para comparação das proporções entre amostras não-pareadas, ou o teste de McNemar para amostras pareadas. O nível de significância estatística para todas as associações em qualquer nível de análise foi definido em 5\%, bicaudal. A análise dos dados foi realizada pelo programa Epi Info, versão 3.3.2.

O estudo foi financiado pela bolsa da Capes e por recursos próprios.

\section{Resultados}

Foram estudados 88 pacientes com idade média de $63,1 \pm 9,9$ anos (tab. 1). Houve maior porcentual de homens, de $58 \%(n=51)$, com idade média de $62,4 \pm 10,5$ anos, e $42 \%$ de mulheres ( $n=37)$, com $64,1 \pm 9,0$ anos; $48,8 \%$ do total de pacientes apresentaram eventos isquêmicos recentes (48,8\%), e $63,6 \%$ eram usuários do SUS. As demais características clínicas e demográficas estão expressas na tabela 1.

A entrevista de seguimento foi realizada em média após 8,8 meses (4,8 - 14,2 meses), mediana 8,4 meses. O número médio de avaliações dos pacientes em consultório após a alta foi de 4,1 $\pm 2,4(0-10)$ mediana de quatro avaliações. No seguimento, todos os pacientes apresentavam pelo menos um fator de risco cardiovascular entre hipertensão, diabete, dislipidemia, antecedentes familiares de coronariopatia, IMC acima de 25, ou obesidade abdominal. Sessenta e nove pacientes $(89,6 \%)$ apresentavam síndrome metabólica; 82 $(93,2 \%)$ utilizavam antiagregantes plaquetários; 61 (69,3\%),

\begin{tabular}{|c|c|c|c|c|}
\hline & $\mathbf{n}$ & $\%$ & Média & DP \\
\hline Idade (anos) & & & 63,1 & 9,9 \\
\hline \multicolumn{5}{|l|}{ Gênero } \\
\hline Masculino & 51 & 58,0 & $62,4^{*}$ & 10,5 \\
\hline Feminino & 37 & 42,0 & $64,1^{*}$ & 9,0 \\
\hline Renda familiar (no salários mínimos) & & & 6,6 & 6,6 \\
\hline \multicolumn{5}{|l|}{ Seguro saúde } \\
\hline SUS & 56 & 63,6 & & \\
\hline Privado & 32 & 36,4 & & \\
\hline \multicolumn{5}{|l|}{ Escolaridade } \\
\hline Analfabeto / $1^{\circ}$ grau incompleto & 47 & 53,4 & & \\
\hline $1^{\circ}$ grau completo $/ 2^{\circ}$ grau incompleto & 7 & 8,0 & & \\
\hline $2^{\circ}$ grau completo $/ 3^{\circ}$ grau incompleto & 23 & 26,1 & & \\
\hline $3^{\circ}$ grau completo ou superior & 11 & 12,5 & & \\
\hline \multicolumn{5}{|l|}{ Diagnóstico que levou ao procedimento } \\
\hline Angina estável & 25 & 31,3 & & \\
\hline Angina instável & 22 & 27,5 & & \\
\hline Infarto agudo do miocárdio & 17 & 21,3 & & \\
\hline Insuficiência cardíaca congestiva & 9 & 11,3 & & \\
\hline Assintomático & 7 & 8,8 & & \\
\hline \multicolumn{5}{|l|}{ Fatores de risco } \\
\hline Hipertensão arterial & 86 & 97,7 & & \\
\hline Diabete melito & 38 & 43,2 & & \\
\hline Dislipidemia & 85 & 96,6 & & \\
\hline Tabagismo & 10 & 11,4 & & \\
\hline História familiar de coronariopatia & 41 & 47,7 & & \\
\hline
\end{tabular}

\footnotetext{
* idade em anos.
} 


\section{Artigo Original}

betabloqueadores; 48 (54,5\%), inibidores da enzima conversora da angiotensina (IECA); 13 (14,8\%), antagonistas dos receptores AT1 da Angiotensina II (antagonistas AII); e 83 $(96,5 \%)$ faziam uso de pelo menos um anti-hipertensivo.

Dentre os dislipidêmicos, 67 (78,8\%) faziam uso de estatinas; e dentre os diabéticos, 35 (92,1\%) utilizavam antidiabéticos orais ou insulina. Em relação ao período pré-operatório, foi observado um acréscimo no uso de estatinas de $60 \%$ (de $47,7 \%$ para $76,1 \%, \mathrm{P}<0,001$ ), e de antiagregantes plaquetários de $24 \%$ (de $75,0 \%$ para $93,2 \%$, $\mathrm{P}<0,001$ ) (Gráfico 1). Não houve diferença significativa entre o período pré e pós-operatório para as demais medicações. A quantificação de variáveis contínuas relacionadas aos FR está evidenciada na tabela 2. A meta de controle da hipertensão foi atingida para 21 pacientes (24,4\%); do colesterol LDL, para 26 pacientes $(30,6 \%)$; da glicemia, para 12 pacientes $(31,6 \%)$ (tab. 3). Trinta pacientes $(36,1 \%)$ encontravam-se na faixa de peso ideal; $38(43,2 \%)$ relatavam prática de exercícios físicos

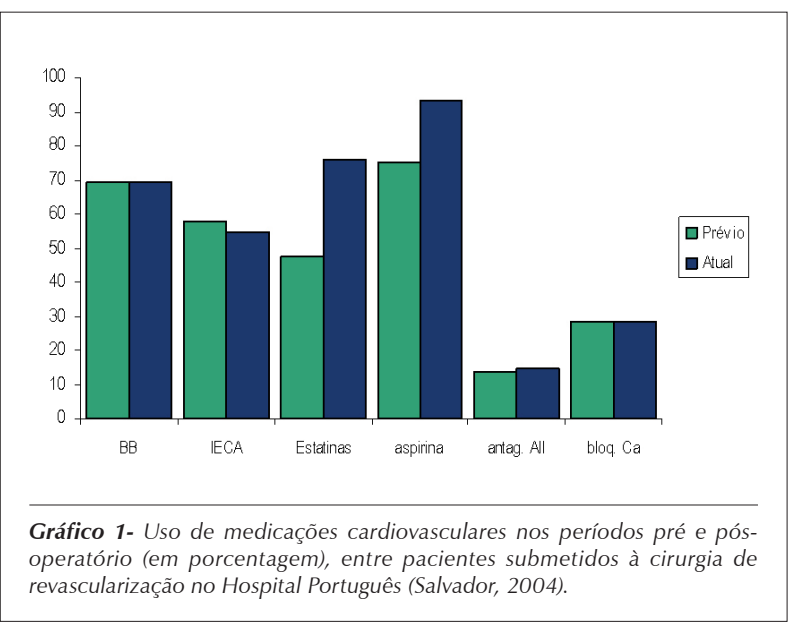

aeróbicos regulares; e entre os tabagistas, 70\% deixaram de fumar. Apenas 14,8\% dos pacientes apresentavam simultaneamente níveis adequados de glicemia, colesterol LDL e pressão arterial.

Não houve diferença significativa entre homens e mulheres em relação a características basais, porém houve associação entre o gênero feminino e menor grau de escolaridade (escolaridade igual ou superior ao segundo grau completo de $27 \%$ versus $47,1 \%, P=0,057)$. Entre os homens, foi evidenciado maior porcentual de pacientes controlados para obesidade abdominal (59,5\% versus 9,7\%, $\mathrm{P}<0,001)$ e para sedentarismo (52,9\% versus $29,7 \%, \mathrm{P}<0,05)$.

Pacientes que possuíam convênios privados de saúde apresentaram renda significativamente maior (mediana de 7,0 versus $2,5, \mathrm{P}<0,001$ ), maior grau de escolaridade (escolaridade igual ou superior ao segundo grau completo $62,5 \%$ versus $25,0 \%, P=0,001)$ e maior controle do colesterol LDL (48,4\% versus 20,4\%, P = 0,025) em relação aos pacientes do SUS. Obesidade foi mais freqüente entre pacientes usuários de convênios privados $(27,6 \%$ versus $7,4 \%, P=0,025)$, porém pacientes atendidos pelo SUS apresentaram maior porcentual de sobrepeso $(57,4 \%$ versus $34,5 \%, P=0,025$ ) (tab. 4). A proporção de pacientes com peso controlado não apresentou diferença significativa entre os dois grupos. Houve uso mais freqüente de IECA entre os pacientes atendidos pelo SUS $(66,1 \%$ versus $34,4 \%, \mathrm{P}=$ $0,004)$. Os pacientes que possuíam convênio privado fizeram uso mais freqüente de estatinas $(87,5 \%$ versus $69,6 \%, \mathrm{P}=$ $0,059)$ e de antagonistas A II $(25,0 \%$ versus $8,9 \%, P=0,06)$, embora essas diferenças não atinjam significância estatística em relação ao valor de p definido pelo estudo.

\section{Discussão}

O presente estudo envolveu pacientes com elevado risco de novos eventos cardiovasculares, pois além de submetidos

\begin{tabular}{|c|c|c|c|c|}
\hline & Média & Mediana & DP & Variação \\
\hline Colesterol total (mg/dl) & 181,5 & 172,0 & 54,8 & $105-355$ \\
\hline Colesterol LDL (mg/dl) & 104,8 & 104,0 & 38,1 & $36-210$ \\
\hline Colesterol HDL (mg/dl) & 43,6 & 43,0 & 11,1 & $10,4-72,0$ \\
\hline Triglicérides (mg/dl) & 164,6 & 138,5 & 102,6 & $58-663$ \\
\hline Glicemia (mg/dl) & 123,0 & 102,8 & 55,5 & $51-338$ \\
\hline Pressão arterial sistólica (mmHg) & 148,6 & 145,0 & 28,7 & $100-260$ \\
\hline Pressão arterial diastólica (mmHg) & 86,7 & 82,0 & 16,3 & $60-140$ \\
\hline Índice de massa corpórea (kg/m²) & 26,3 & 26,0 & 3,8 & $19,2-38,1$ \\
\hline Circunferência abdominal $(\mathrm{cm})$ & 99,2 & 98,0 & 9,9 & $77-121$ \\
\hline Homens & 100,0 & 100,2 & 10,1 & $77-120$ \\
\hline Mulheres & 98,0 & 96,0 & 9,7 & $80-121$ \\
\hline
\end{tabular}




\begin{tabular}{|c|c|c|}
\hline & $\mathbf{n}$ & $\%$ \\
\hline \multicolumn{3}{|c|}{ Controle de fatores de risco cardiovascular } \\
\hline Tabagismo & $7 / 10$ & 70 \\
\hline Sedentarismo & $38 / 88$ & 43,2 \\
\hline Obesidade abdominal* & $8 / 73$ & 11,0 \\
\hline $\mathrm{IMC} \leq 25 \mathrm{~kg} / \mathrm{m}^{2}$ & $30 / 83$ & 36,1 \\
\hline Diabete melito & $12 / 38$ & 31,6 \\
\hline Hipertensão & $21 / 86$ & 24,4 \\
\hline \multicolumn{3}{|l|}{ Perfil lipídico desejável } \\
\hline $\mathrm{CT}<200 \mathrm{mg} / \mathrm{dl}$ & $42 / 85$ & 49,4 \\
\hline $\mathrm{LDL}<100 \mathrm{mg} / \mathrm{dl}$ & $26 / 85$ & 30,6 \\
\hline Triglicérides < 150 mg/dl & $32 / 86$ & 37,2 \\
\hline $\mathrm{HDL}>40 \mathrm{mg} / \mathrm{dl}(45 \mathrm{mg} / \mathrm{dl}$ se DM) & 29/86 & 33,7 \\
\hline
\end{tabular}

FR - fatores de risco; DM - diabete melito; HAS - hipertensão arterial sistêmica; CT - colesterol total; LDL - low density lipoprotein; HDL - high density lipoprotein; * Utilizados os critérios de obesidade abdominal para a América do Sul (IDF, 2006).

Tabela 4 - Frequêência de fatores de risco entre pacientes usuários do SUS e conveniados (Salvador, Bahia)

\begin{tabular}{lcc} 
& SUS $(\mathbf{n = 5 6 )}$ & Convênio $(\mathbf{n}=\mathbf{3 2})$ \\
Hipertensão & $55(98,2 \%)$ & $31(96,9 \%)$ \\
Diabete melito & $23(41,1 \%)$ & $15(46,9 \%)$ \\
Dislipidemia & $54(96,4 \%)$ & $31(96,9 \%)$ \\
Tabagismo & $6(10,7 \%)$ & $4(12,5 \%)$ \\
Obesidade & $4(7,4 \%)$ & $8(27,6 \%)$ \\
Sobrepeso & $31(57,4 \%)$ & $10(34,5 \%)$ \\
Obesidade abdominal * & $28(62,2 \%)$ & $17(60,7 \%)$ \\
\hline
\end{tabular}

* De acordo com os critérios da International Diabetes Federation para a América do Sul (IDF, 2006).

à cirurgia de RM, 48,8\% já haviam apresentado eventos isquêmicos recentes, e 11,3\% apresentavam insuficiência cardíaca esquerda. Apesar disso, um elevado porcentual de pacientes não atingiu um controle adequado de FR modificáveis, como hipertensão, diabete e hipercolesterolemia, após pelo menos seis meses da realização de cirurgia de RM. Nesse estudo, apenas 24,4\% dos hipertensos estavam controlados a despeito do uso regular de pelo menos um agente anti-hipertensivo. Para diabete e dislipidemia, os níveis de controle foram de $31,6 \%$ e $30,6 \%$, respectivamente. Isso é especialmente relevante quando consideramos a elevada prevalência de hipertensão, diabete e dislipidemia nesse estudo, que foi $97,7 \%, 43,2 \%$ e $96,6 \%$, respectivamente.

É importante salientar que nesse estudo houve elevada utilização de medicações quando comparado a outros estudos, chegando a $96,5 \%, 92,1 \%$ e $78,8 \%$ de tratamento com antihipertensivos, hipoglicemiantes e estatinas, respectivamente. Curiosamente, apesar do uso de tratamento farmacológico considerado eficaz para o controle dos principais FR (hipertensão, diabete e dislipidemia), isso ocorreu em apenas um pequeno número de pacientes. Em estudo realizado na Turquia envolvendo pacientes revascularizados, apenas 24,2\% dos pacientes fizeram uso de agentes redutores do colesterol, e $62,6 \%$ fizeram tratamento anti-hipertensivo ${ }^{12}$.

Os pacientes estudados, na sua maioria, apresentavam baixos níveis de escolaridade, com mais de 50\% declarando-se analfabetos ou com primeiro grau incompleto, e cuja mediana de renda foi de quatro salários mínimos. Aproximadamente $36 \%$ dos pacientes possuíam seguro privado de saúde. Esses pacientes apresentavam maior renda familiar e maior grau de escolaridade, o que propicia maiores condições econômicas para adquirir medicações mais caras, como as estatinas e os antagonistas All, além de maior esclarecimento em relação à sua doença e quanto à necessidade de medidas preventivas. Esses mesmos fatores podem estar influenciando no maior controle de obesidade abdominal e sedentarismo entre os homens, dado que esses apresentavam maior grau de escolaridade. A diferença observada em relação ao uso de 
IECA pode ser explicada pelo fato de essa medicação ser fornecida gratuitamente aos usuários do SUS nos postos de saúde. Outro ponto interessante é que pacientes conveniados fizeram uso mais freqüente de outras classes de antihipertensivos como os antagonistas A II.

Os pacientes realizaram, em média, quatro consultas no período de seguimento, isto é, em média uma consulta a cada dois meses, número superior ao preconizado pelas diretrizes brasileiras de cirurgia de revascularização ${ }^{13}$. A realização desse número de consultas, associada à elevada freqüência do uso de fármacos no pós-operatório sem um controle satisfatório, pode sugerir que medidas de abordagem mais qualitativas sejam necessárias para entender e corrigir os eventos que estão de fato ocorrendo.

Apesar de realizar reduzido número de cirurgias, bem abaixo da sua necessidade, o Estado da Bahia representa o 14 o colocado em todo o Brasil e o quarto colocado na Região Nordeste em relação ao número de cirurgias realizadas pelo SUS ${ }^{14}$. Os procedimentos que foram objeto desse estudo corresponderam a 42,1\% do total de cirurgias de RM realizadas nos pacientes do SUS na Bahia, no ano de 2004 (133 procedimentos). Portanto, apesar de o estudo ter sido realizado em apenas um hospital da Bahia, o número de pacientes operados correspondeu à expressiva parcela de pacientes submetidos a cirurgia de RM em nosso Estado. Os resultados encontrados muito provavelmente podem ser generalizados, uma vez que as condições de acesso aos serviços de saúde para a realização desse tipo de procedimento são bastante semelhantes em todos os Estados do Nordeste, e quiçá, em vários Estados do Brasil, além do fato de que os resultados encontrados estão em conformidade com estudos prévios realizados na Bahia e em outras partes do mundo ${ }^{5,7,15}$.

Em estudo realizado em Salvador envolvendo 104 pacientes com DAC, foi observada menor prevalência de hipertensão $(67,3 \%)$ e diabete $(20,2 \%)$, mas níveis médios de colesterol total $(222,9 \mathrm{mg} / \mathrm{dl})$ e de colesterol LDL (144,9 $\mathrm{mg} / \mathrm{dl}$ ) bastante acima dos limites considerados aceitáveis ${ }^{15}$. Foi evidenciado controle de hipertensão arterial (PA $<140 \times 90$ $\mathrm{mmHg}$ ) em $42,2 \%$ dos pacientes, do excesso de peso (IMC $<25 \mathrm{~kg} / \mathrm{m}^{2}$ ) em 37,6\%, de hipercolesterolemia (CT < 200 $\mathrm{mg} / \mathrm{dl}$ ) em 31,8\% e do colesterol LDL (inferior a $100 \mathrm{mg} / \mathrm{dl}$ ) em $8,1 \%$. Contudo, esses dados foram coletados antes da publicação das últimas recomendações para o controle do colesterol, em que os limites considerados aceitáveis para o seu controle foram reduzidos ${ }^{10}$.

Recentemente foi realizado grande estudo multicêntrico, o EUROASPIRE II, com o intuito de verificar se houve melhora na prática de medidas preventivas após a publicação de diretrizes que preconizavam pontos de corte menores para o controle de FR7 . Segundo esse estudo, que envolveu 5.556 pacientes portadores de DAC (isquemia miocárdica, infarto agudo do miocárdio, ou após realização de angioplastia ou cirurgia de RM) em 15 países europeus, também se pôde observar elevada prevalência de FR não-controlados após 1,4 ano de seguimento. Após esse período, observou-se elevada taxa de obesidade $(31,3 \%)$ e obesidade abdominal (79,8\%); 49,5\% dos pacientes apresentavam níveis pressóricos controlados (< $140 \times 90 \mathrm{mmHg}) ; 41,7 \%$ apresentavam níveis de colesterol total abaixo de 200 mg/dl; e 13\% níveis glicêmicos abaixo de 110 mg/dl. Entre os pacientes tabagistas, $34,4 \%$ deixaram de fumar. Também foi evidenciado baixo uso de agentes profiláticos, com $86 \%$ de uso de AAS, $63 \%$ de betabloqueadores, 38\% de IECA e 55,3\% de estatinas.

Outro estudo realizado em centro de cirurgia cardíaca do Reino Unido envolvendo 214 pacientes revascularizados também evidenciou controle inadequado de FR após um ano de seguimento, inclusive com piora do controle pressórico quando comparado ao período pré-operatório, com níveis de PAS $\geq 140 \mathrm{mmHg}$ passando de $40,1 \%$ para $60 \%$, e de PAD $\geq$ $90 \mathrm{mmHg}$ de $30 \%$ para $43 \%$, com significativa diminuição do uso de importantes medicações, como AAS, que passou de $80,2 \%$ para $69,2 \%$, e betabloqueadores, de $58,5 \%$ para $17 \%{ }^{16}$.

Nos últimos anos, tem-se observado grande esforço por parte das sociedades médicas no sentido de melhorar o controle de $\mathrm{FR}^{17-20}$. Apesar da normatização, as dificuldades para alcançar adequado controle desses FR persistem. Alguns estudos sugerem que ações coordenadas em forma de programas de reabilitação, com ênfase em ações que envolvem aspectos psicológicos e sociais, podem ser mais eficientes que ações isoladas ${ }^{21-22}$. Essa percepção mais abrangente no pós-operatório da cirurgia de RM pode ser vista nas recomendações da World Health Organization, que recomenda programas de reabilitação cardíaca definidos como "a soma de atividades necessárias para assegurar as melhores condições físicas, mentais e sociais possíveis, de forma a que o paciente cardíaco possa retornar, o tanto quanto possível, ao seu lugar na vida da comunidade"23.

Outro ponto a ser salientado é que, em paralelo ao baixo controle de FR, constatou-se um número apreciável de pacientes sem exames recentes para avaliação do controle do colesterol LDL (35,4\% do total) e da glicemia (13,2\%), o que sugere que os pacientes podem estar encontrando dificuldades para ter acesso a orientações médicas e para realizar exames. Ou seja, pouco apoio para implementar mudanças de hábitos tão importantes, como alterar a dieta, fazer atividade física, abandonar o tabagismo e etilismo, e principalmente se dedicar a hábitos de vida mais saudáveis. Esses dados sugerem que pacientes graves estão conseguindo ter acesso a serviços complexos da rede hospitalar em tempo hábil, o que permite a execução da cirurgia e a sobrevivência, porém a continuidade desses cuidados na fase de acompanhamento ambulatorial é insuficiente, como pode ser visto pelos baixos índices de controle de FR mostrados nesse estudo.

Algumas medidas poderiam ser tomadas com o intuito de melhorar o controle de FR, como o fornecimento regular e mais amplo das medicações de forma gratuita nos postos de saúde, particularmente aquelas de custo mais elevado, como as estatinas, além da realização de exames complementares, como avaliação de perfil lipídico, glicemia e hemoglobina glicosilada nos próprios postos de saúde. O acompanhamento multidisciplinar por meio de programas de reabilitação implantados pelo governo, seguindo as recomendações preconizadas pelas diretrizes, também poderia ser de grande auxílio para atingir esse objetivo.

A principal limitação do estudo consiste no fato de envolver apenas pacientes submetidos a cirurgia de RM em um único hospital da Bahia. Porém, isso é amenizado quando observamos que o número de pacientes operados correspondeu a uma 
parcela expressiva dos pacientes submetidos a cirurgia de RM em nosso Estado, como já referido. Além disso, naquele ano apenas dois outros hospitais da cidade atendiam pacientes do SUS que necessitassem realizar procedimentos de RM. Outra possível limitação seria o uso da glicemia de jejum para avaliar controle do diabete, em vez da glicemia pós-prandial ou da $\mathrm{Hb}$ glicosilada. Contudo, de acordo com as diretrizes da IDF, essa é uma estratégia possível em estruturas que só disponham de cuidados mínimos, como parece ser nossa situação atual.

Alguns pacientes revascularizados não puderam comparecer à entrevista, seja por falta de recursos seja por dificuldades para a sua localização por conta de informações insuficientes e registros errados de telefone e endereço. Contudo, a maioria desses pacientes era residente no interior, usuários do SUS, o que torna improvável que estivessem recebendo cuidados melhores que os pacientes do estudo. No momento da entrevista os pacientes foram questionados em relação às medicações que vinham em uso, mas não foi utilizado nenhum método sistematizado para a avaliação da adesão ao tratamento, como a contagem das embalagens vazias. Não foi feito o registro das doses das medicações utilizadas, de forma que não podemos avaliar se os objetivos de controle não foram atingidos por causa do uso de doses inadequadas das medicações. Os pacientes não foram avaliados em relação ao controle de FR antes do procedimento cirúrgico, haja vista que o estudo começa no momento da internação para cirurgia.

\section{Referências}

1. Rogers WJ, Coggin CJ, Gersh BJ, Fisher LD, Myers WO, Oberman A, et al. Ten-year follow-up of quality of life in patients randomized to receive medical therapy or coronary artery bypass graft surgery. The Coronary Artery Surgery Study (CASS). Circulation. 1990; 82 (5): 1859-62.

2. Glueck, C.J. Role of risk factor management in progression and regression of coronary and femoral artery atherosclerosis. Am J Cardiol. 1986; 57 (Supl G): $35-41$.

3. Corti R, Fayad ZA, Fuster V, Worthley SG, Helft G, Chesebro J, et al. Effects of lipid-lowering by simvastatin on human atherosclerotic lesions: a longitudinal study by high-resolution, noninvasive magnetic resonance imaging. Circulation. 2001; 104 (3): 249-52.

4. White, C.W. Benefit of aggressive lipid-lowering therapy: insights from the post coronary artery bypass graft study and other trials. Am J Med. 1998; 105 (Suppl 1A): 63-8.

5. Euroaspire Study Group. A European Society of Cardiology survey of secondary prevention of coronary heart disease: principal results. EUROASPIRE Study Group. European Action on Secondary Prevention through Intervention to Reduce Events. Eur Heart J. 1997; 18 (10): 156982.

6. Wood D, De Backer G, Faergeman O, Graham I, Mancia G, Pyorala K. Prevention of coronary heart disease in clinical practice: recommendations of the Second Joint Task Force of European and other Societies on Coronary Prevention. Atherosclerosis. 1998; 140 (2): 199-270.

7. Euroaspire II study group. Lifestyle and risk factor management and use of drug therapies in coronary patients from 15 countries: principal results from Euroaspire II Euro Heart Survey Programme. Eur Heart J. 2001; 22 (7): 554-72.

8. Sociedade Brasileira de Cardiologia, Sociedade Brasileira de Hipertensão, Sociedade Brasileira de Nefrologia. V Diretrizes Brasileiras de Hipertensão Arterial. Rev Bras Hipertens. 2006; 13 (4): 256-312.

9. Sociedade Brasileira de Hipertensão, Sociedade Brasileira de Cardiologia,

\section{Conclusões}

O controle de fatores de risco cardiovascular tem sido realizado de forma insuficiente entre pacientes portadores de doença aterosclerótica coronariana que foram submetidos a cirurgia de revascularização miocárdica.

Apesar do uso freqüente de medicações para o controle de hipertensão, diabete e hipercolesterolemia pela maioria dos pacientes, isso não resultou diretamente em adequado controle dos principais fatores de risco cardiovascular, sugerindo que existe grande espaço para a melhoria da atenção médica preventiva dirigida a esse grupo de pacientes.

\section{Potencial Conflito de Interesses}

Declaro não haver conflito de interesses pertinentes.

\section{Fontes de Financiamento}

O presente estudo foi parcialmente financiado por Bolsa de Mestrado da CAPES .

\section{Vinculação Acadêmica}

Este artigo é parte de tese de mestrado de Andrea Cristina Costa Barbosa, Luiz Carlos Santana Passos, Antônio Alberto Lopes, Wanewman Lins Guedes de Andrade, Luciano Rapold Souza, Maurício Batista Nunes pela Universidade Federal da Bahia e Hospital Português.

Sociedade Brasileira de Endocrinologia e Metabologia, Sociedade Brasileira de Diabetes, Associação Brasileira para Estudos da Obesidade. I Diretriz Brasileira de Diagnóstico e Tratamento da Síndrome Metabólica. Arq Bras Cardiol. 2005, 84 (Supl I): 1-28.

10. Sociedade Brasileira de Cardiologia. III Diretrizes Brasileiras Sobre Dislipidemias e Diretriz de Prevenção da Aterosclerose do Departamento de Aterosclerose da Sociedade Brasileira de Cardiologia. Arq Bras Cardiol. 2001; 77 (Supl III): 1-48.

11. International Diabetes Federation [homepage na internet]. Diabetes Prevalence [citado 2005 setembro 10]. Disponível em: http://www.idf. org/home/index.cfm?node $=264$.

12. Baltali M, Kiziltan HT, Korkmaz ME, Topcu S, Demirtas M, Metin M, et al. Prevalence of modifiable cardiovascular risk factors remain high after coronary bypass graft surgery: a multicentre study among Turkish patients. J Cardiovasc Risk. 2002; 9 (4): 207-14.

13. Sociedade Brasileira de Cardiologia. Diretrizes de cirurgia de revascularização miocárdica, valvopatias e doenças da aorta. Arq Bras Cardiol. 2004; 82 (supl V): 1-21.

14. Ministério da Saúde. Secretaria Executiva. Datasus, Sistema de Informações Hospitalares do SUS [citado 2005 maio 15]. Disponível em: http:// w.w.w.tabnet.datasus.gov.br/tabnet/tabnet.htm.

15. Ladeia AM, Guimarães A.C. Assessment of risk factors in coronary patients being followed by cardiologists: control of risk factors in coronary patients. Prev Cardiol. 2003; 6 (3): 122-7.

16. Belcher PR, Gaw A, Cooper M, Brown M, Wheatley DJ, Lindsay GM. Are we negating the benefits of $\mathrm{CABG}$ by forgetting secondary prevention? J Hum Hypertens. 2002; 16 (10): 691-7.

17. Wood D, De Backer G, Faergeman O, Graham I, Mancia G, Pyorala K, et al. Prevention of coronary heart disease in clinical practice: recommendations of the Second Joint Task Force of European and other Societies on Coronary Prevention. Atherosclerosis. 1998; 140 (2): 199-270. 


\section{Artigo Original}

18. Working Group on Rehabilitation of The European Society of Cardiology Long-term comprehensive care of cardiac patients. Eur Heart J. 1992; 13 (Suppl C): 1-45.

19. British Cardiac Society, British Hyperlipidaemia Association, British Hypertension Society, endorsed by the British Diabetic Association. Joint British recommendations on prevention of coronary heart disease in clinical practice. Heart. 1998; 80(Suppl 2): 1-29.

20. De Backer G, Ambrosioni E, Borch-Johnsen K, Brotons C, Cifkova R, Dallongeville J, et al. European guidelines on cardiovascular disease prevention in clinical practice. Third Joint Task Force Of European and other societies on cardiovascular disease prevention in clinical practice. Eur Heart J. $2003 ; 24$ (17): 1601-10.
21. Lisspers J, Hofman-Bang C, Nordlander R, Ryden L, Sundin O, Ohman A, et al. Multifactorial evaluation of a program for lifestyle behavior change in rehabilitation and secondary prevention of coronary artery disease. Scand Cardiovasc J. 1999; 33 (1): 9-16.

22. Dalal HM, Evans PH. Achieving national service framework standards for cardiac rehabilitation and secondary prevention. BMJ. 2003; 326 (7387): 481-4.

23. Hedback B, Perk J, Hornblad M, Ohlsson U. Cardiac rehabilitation after coronary artery bypass surgery: 10-year results on mortality, morbidity and readmissions to hospital. J Cardiovasc Risk. 2001; 8 (3): 153-8. 